\title{
Giant Plasticity of a Quantum Crystal
}

\author{
Ariel Haziot, ${ }^{1}$ Xavier Rojas, ${ }^{1}$ Andrew D. Fefferman, ${ }^{1}$ John R. Beamish, ${ }^{1,2}$ and Sébastien Balibar ${ }^{1}$ \\ ${ }^{1}$ Laboratoire de Physique Statistique de l'Ecole Normale Supérieure, associé au CNRS et aux Universités P.M. Curie \\ and D. Diderot, 24 rue Lhomond, 75231 Paris Cedex 05, France \\ ${ }^{2}$ Departement of Physics, University of Alberta, Edmonton, Alberta Canada T6G $2 G 7$
}

(Received 12 October 2012; published 14 January 2013)

\begin{abstract}
When submitted to large stresses at high temperature, usual crystals may irreversibly deform. This phenomenon is known as plasticity and it is due to the motion of crystal defects such as dislocations. We have discovered that, in the absence of impurities and in the zero temperature limit, helium 4 crystals present a giant plasticity that is anisotropic and reversible. Direct measurements on oriented single crystals show that their resistance to shear nearly vanishes in one particular direction because dislocations glide freely parallel to the basal planes of the hexagonal structure. This plasticity disappears as soon as traces of helium 3 impurities bind to the dislocations or if their motion is damped by collisions with thermal phonons.
\end{abstract}

A crystal resists elastically to a shear stress, contrary to a fluid, which flows. When applied to a crystal, a small shear stress $\sigma$ produces a strain $\varepsilon$ that is proportional to $\sigma$. If the stress is released, the crystal returns elastically to its original shape. The elastic shear modulus of the crystal is the ratio $\mu=\sigma / \varepsilon$. However, if the applied stress is large, a usual crystal is "plastic"-it deforms more than in the elastic regime thanks to the motion of dislocations. With classical crystals, plastic deformation is irreversible and small. In metals, it requires stresses of order $10^{-1}$ to $10^{-6}$ of the shear modulus and the strain rate increases with temperature [1,2]. We have discovered that, in the case of ultrapure helium 4 single crystals around $0.1 \mathrm{~K}$, the resistance to shear nearly vanishes in one particular direction while in another direction no measurable deviation from normal elastic behavior occurs. This giant anisotropic plasticity is independent of stress down to extremely low values $\left(10^{-11}\right.$ times the elastic shear modulus). We demonstrate that it is a consequence of dislocations gliding freely along the basal planes of the hexagonal crystal structure. Some gliding along these planes had been observed $[3,4]$ in other hexagonal crystals but never with comparable amplitude. In ${ }^{4} \mathrm{He}$ crystals, we show that dislocations are able to move at high speed with negligible dissipation so that the plasticity is also independent of the rate at which the strain is applied. Moreover, this motion is reversible so that the plasticity shows up as a $50 \%$ to $80 \%$ reduction of one particular elastic coefficient, which we have identified as $c_{44}$. The plasticity disappears if traces of impurities bind to dislocations or if thermal phonons damp their motion. Our observations have been made possible by direct elasticity measurements on oriented crystals where the impurity concentration could be lowered from $0.3 \mathrm{ppm}$ to zero at very low temperature $(15 \mathrm{mK})$. The gliding of dislocations is an intensively studied phenomenon of fundamental importance in materials science [5]. We provide strong experimental evidence for its precise origin.

We have improved some of our previous techniques [6-9]. Here we briefly describe them (see details in the Supplemental Material [10]). In a transparent cell inside a dilution refrigerator with optical access, crystals can be seen during growth. After determining their orientation from their shape (Fig. 1), we grow them in a $1 \mathrm{~mm}$ gap between two piezoelectric transducers. Applying a small ac voltage to one transducer ( 0.001 to $1 \mathrm{~V}, 200$ to $20000 \mathrm{~Hz})$ produces a vertical displacement $(0.001$ to $1 \AA$ ) and consequently a strain $\varepsilon$ of amplitude $10^{-10}$ to $10^{-7}$ and a stress $\sigma=\mu \varepsilon\left(10^{-9}\right.$ to $10^{-5}$ bar $)$ on the other transducer ( $\mu$ is the relevant shear modulus of the

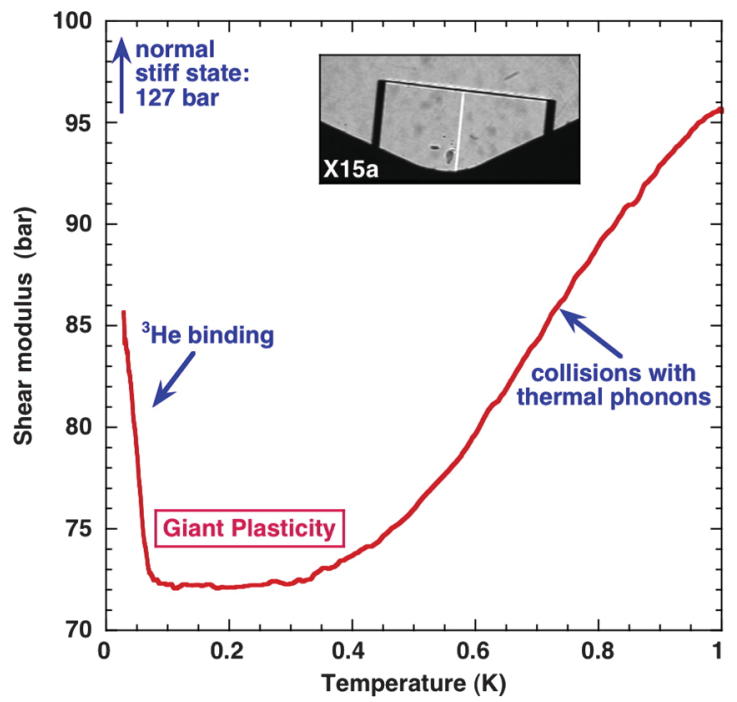

FIG. 1 (color). The shear modulus of crystal X15a as a function of temperature. Around $0.2 \mathrm{~K}$, the shear modulus is reduced to $72 \mathrm{bar}$, much less than the normal value (127 bar). 
crystal). The stress generates a current that is our signal after amplification with a lock-in. The shear modulus $\mu$ and the dissipation $1 / Q$ are obtained from the magnitude and phase of the signal. Various growth methods lead to three different types of crystals [7]:

(i) High quality "type 1" crystals are grown by pressurizing the superfluid liquid in the cell at $20 \mathrm{mK}$ up to the crystallization pressure and staying there after the cell is nearly full. Random nucleation produces different orientations. In this case, there remains some liquid in corners but not in the gap. Fresh from growth, such crystals are totally free of impurities, especially if one uses ultrapure ${ }^{4} \mathrm{He}$ containing $0.4 \mathrm{ppb}$ of ${ }^{3} \mathrm{He}$ (we also use natural ${ }^{4} \mathrm{He}$ with $0.3 \mathrm{ppm}$ ${ }^{3} \mathrm{He}$ ). Slow growth at $20 \mathrm{mK}$ rejects ${ }^{3} \mathrm{He}$ impurities in front of the liquid-solid interface as in the "zone melting" purification of metals but here this method is extremely efficient because the temperature is very low [8]. However, when warming such ultrapure crystals, ${ }^{3} \mathrm{He}$ impurities come back into the crystal where their concentration varies with temperature.

(ii) Single crystals of lower quality (type 2) are grown at $1.4 \mathrm{~K}$. All the remaining liquid is crystallized during further cooling along the melting line from 26.1 bar at $1.4 \mathrm{~K}$ to 25.3 bar at $1 \mathrm{~K}$, below which it stays constant [11]. This pressure change probably creates dislocations, but the ${ }^{3} \mathrm{He}$ concentration is that of the helium gas used to grow them. Any type 1 crystal can be melted down to a small seed and regrown at $1.4 \mathrm{~K}$ as a type 2 crystal with the same orientation, or vice versa.

(iii) Polycrystals (type 3) are grown at constant volume starting with normal liquid at $3 \mathrm{~K}$ and 60 bar.

Figure 1 presents a measurement of the shear modulus $\mu$ of crystal X15a at $9 \mathrm{kHz}$ during cooling. This is an ultrapure type 1 crystal, although grown at $600 \mathrm{mK}$. Its shear modulus reaches a very small value around $0.2 \mathrm{~K}$. At lower $T$, the crystal stiffens as a consequence of dislocation pinning by ${ }^{3} \mathrm{He}[6,9,12]$. Crystals with immobile dislocations are stiffer than with mobile dislocations. The pinning temperature depends on the ${ }^{3} \mathrm{He}$ concentration, on the binding energy $E_{3}=0.73 \mathrm{~K}$ [12], and on the measurement frequency. Above $0.3 \mathrm{~K}, \mu$ also increases, probably because dislocations scatter thermal phonons [13]. We thus understand that ${ }^{4} \mathrm{He}$ crystals show an anomalous shear modulus between impurity pinning at very low $T$ and collisions with thermal phonons at higher $T$. We call it a "giant plasticity" because it is due to the large motion of dislocations under very small stresses. Since it is reversible, the plasticity shows up as a reduction in the shear modulus. Note that the present measurements are made at $\mathrm{kHz}$ frequencies during hours with results that are independent of the sign and duration of the applied strain.

Two type 2 crystals are compared in Fig. 2(a): X15c (purity $0.4 \mathrm{ppb})$ and $\mathrm{X} 21\left(0.3 \mathrm{ppm}{ }^{3} \mathrm{He}\right)$, at high or

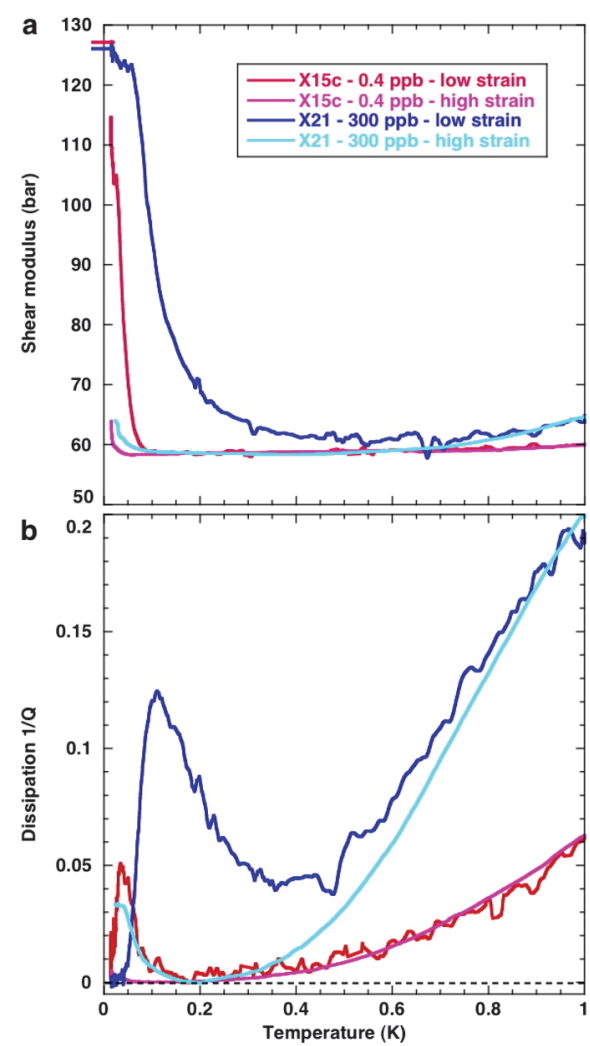

FIG. 2 (color). Comparison of two "type 2" crystals: X15c and X21 grown, respectively, from ultrapure and from natural ${ }^{4} \mathrm{He}$. The $T$ domain of the giant plasticity depends on both purity and strain amplitude. On graph (a), two ticks on the vertical axis indicate the predicted value of the shear modulus for immobile dislocations. The lower graph (b) shows that the dissipation is negligible in the high plasticity region of X15, as for immobile dislocations in the zero temperature limit of X21.

low driving strain. When a larger drive is applied, the temperature at which ${ }^{3} \mathrm{He}$ bind to dislocations is lower, so that the plasticity domain is wider. As expected $[6,9,12]$, pinning by ${ }^{3} \mathrm{He}$ occurs at lower $T$ for ultrapure crystals. Figure 2(b) shows the corresponding dissipation. In the plasticity domain of $\mathrm{X} 15 \mathrm{c}$, we measure zero dissipation, as in the low $T$ limit of $\mathrm{X} 21$ where ${ }^{3} \mathrm{He}$ binding suppresses dislocation motion. The peak dissipation corresponding to ${ }^{3} \mathrm{He}$ binding is lower in temperature for the ultrapure crystal $\mathrm{X} 15 \mathrm{c}$ and it nearly disappears at a high strain $\varepsilon=10^{-7}$ that exceeds the threshold for ${ }^{3} \mathrm{He}$ unbinding $\left(3 \times 10^{-8}\right.$, see Refs. [6,14]). The dissipation above $0.3 \mathrm{~K}$ is independent of amplitude and increases with $T$ as expected for a thermal phonon scattering mechanism [13].

We have identified the precise origin of the plasticity with the following quantitative study. Hexagonal crystals have 5 independent elastic coefficients [14-16] $c_{11}, c_{12}$, $c_{13}, c_{33}, c_{44}$, and $c_{66}=\left(c_{11}-c_{12}\right) / 2$. The indices 1 to 6 , respectively, refer to $x x, y y, z z, y z, x z$, and $x y$, where $z$ is the [0001] " $c$ " axis. Figure 3 shows results from 7 type 2 crystals, all of natural purity except the ultrapure $\mathrm{X} 15$, and one polycrystal (BC2). $\mathrm{X} 3$ is tilted with a 


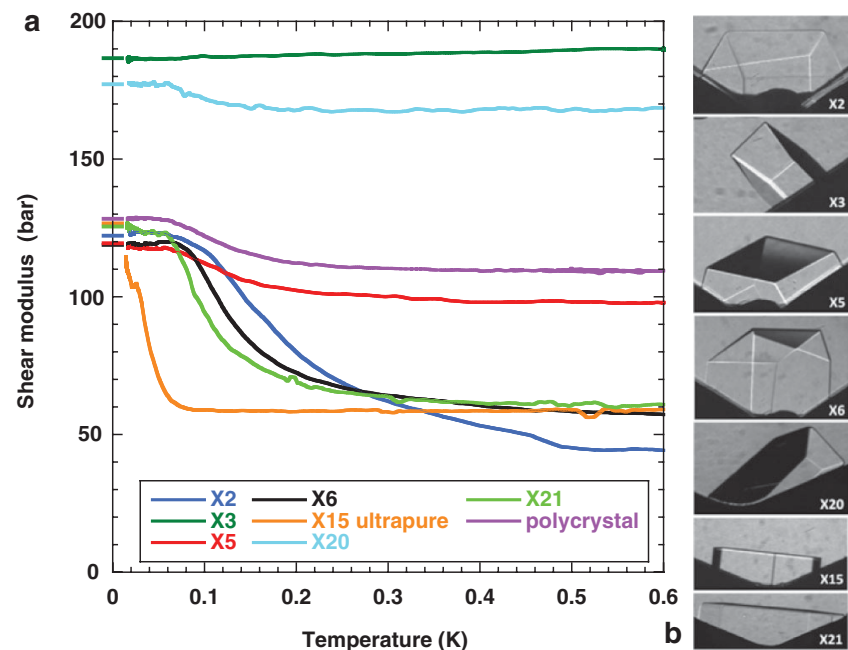

FIG. 3 (color). Measurements (a) for different crystal orientations (b) show that the plasticity is highly anisotropic. Ticks on the vertical axis show the predicted shear modulus with immobile dislocations, in excellent agreement with measurements in the low temperature limit where dislocations are pinned by ${ }^{3} \mathrm{He}$.

$c$ axis $45^{\circ}$ from the vertical. Its effective shear modulus is $\mu=0.004 c_{44}+0.004 c_{66}+0.25\left(c_{11}+c_{33}-2 c_{13}\right)$, practically independent of $c_{44}$ and $c_{66}$ (see the Supplemental Material [10]). It shows no $T$ dependence. Since it is very unlikely that these coefficients would vary with $T$ in just such a way to keep $\left(c_{11}+c_{33}-2 c_{13}\right)$ constant, we conclude that $c_{12}, c_{13}$, and $c_{33}$ are independent of $T$, so that X3 could be used to calibrate the piezoelectric coefficient of our transducers $0.88 \AA \mathrm{A} / \mathrm{V}$ in our first cell, 0.95 in the second cell). For this we used values of elastic coefficients measured from ultrasound velocity at the melting pressure $[15,16] c_{11}=405 \mathrm{bar}, c_{12}=213 \mathrm{bar}, c_{13}=$ 105 bar, $c_{33}=554$ bar, $c_{44}=124$ bar, and $c_{66}=96$ bar. These values correspond to the intrinsic elasticity of ${ }^{4} \mathrm{He}$ crystals because dislocations could not move at $10 \mathrm{MHz}$ above $1.2 \mathrm{~K}$. Given this calibration we could calculate the shear modulus for all other orientations in the $T=0$ limit (ticks on the vertical axis of Fig. 3). Excellent agreement is found with our measurements except for X15 whose stiffening was not completed at $15 \mathrm{mK}$. For the polycrystal BC2, Maris' averaging method [17] leads to excellent agreement again.

All crystals except X3 show a strong $T$ dependence, which could be due to a reduction of either $c_{44}$ or $c_{66}$. Indeed, hexagonal crystals usually have one preferential gliding plane that can be either the basal plane (0001) or the prismatic planes perpendicular to it. For hcp crystals, it is predicted to be the basal plane, although directional bonding in hexagonal metals can lead to non-close-packed structures which favor prismatic glide $[3,4]$. For X21, the dependence on $c_{66}$ is negligible and the large variation of $c_{44}$ could be easily extracted. Assuming then that $c_{66}$ is constant, we could calculate the variation of $c_{44}$ in all

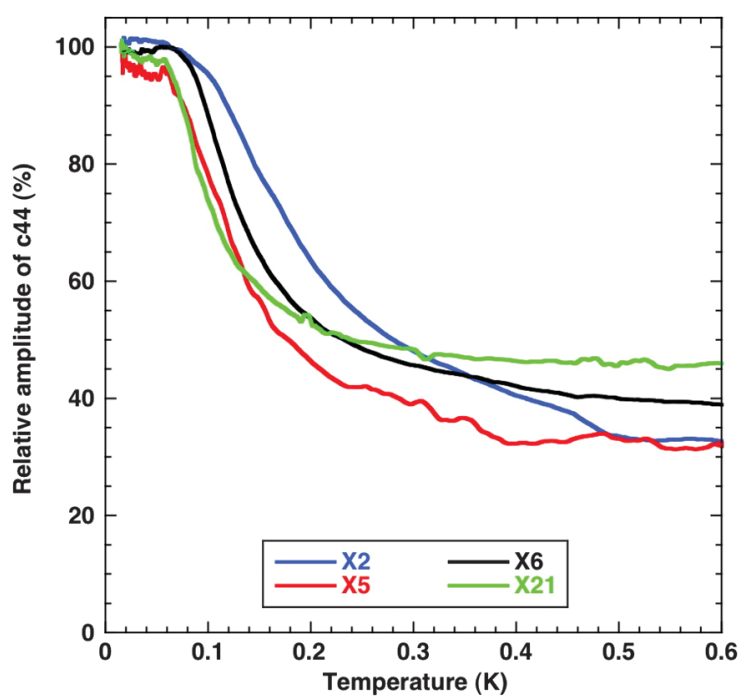

FIG. 4 (color). Assuming that dislocations glide in the basal plane, one finds the same variation of $c_{44}$ by $62 \pm 8 \%$ for $\mathrm{X} 2$, $\mathrm{X} 5, \mathrm{X} 6$, and $\mathrm{X} 21$, which are crystals with different orientations grown in the same conditions. Assuming that they glide in the prismatic planes leads to absurd results (see text).

crystals. Since X2, X5, X6, and X21 were all type 2 crystals grown in similar conditions, one expects the $T$ dependence of their coefficient $c_{44}$ to be the same. Figure 4 shows that this assumption leads to a reproducible reduction of $c_{44}$ ( $62 \pm 8 \%$ for all type 2 crystals), although the shear modulus of $\mathrm{X} 2$ depends mostly on $c_{44}$ while that of X5 depends mostly on $c_{66}$. On the opposite, assuming that $c_{44}$ is constant and $c_{66}$ varies leads to absurd results, from a $60 \%$ reduction in $c_{66}$ for X5 to $300 \%$ for X6 and to $1000 \%$ for X2, not mentioning X21. The preferential direction for gliding has to be the basal plane. Only $c_{44}$ depends on temperature. This is why Fig. 3 shows a highly anisotropic plasticity.

The amplitude dependence is also consistent with ${ }^{3} \mathrm{He}$ pinning at low $T$. Figure 5 shows the shear modulus as a function of stress for 4 crystals at $20 \mathrm{mK}$. Above a $1 \mu$ bar threshold due to the breakaway from ${ }^{3} \mathrm{He}$ impurities [6,14], the shear modulus decreases. By projecting the stress on the basal plane, we determined the "resolved stress" (see Supplemental Material [10]), and we obtained a reproducible threshold for different orientations, confirming that dislocations glide in the basal planes. This threshold is hysteretic because the force acting on dislocations depends on the pinning length $L_{i}$ between impurities [18]. One now understands why Fig. 2 shows a ${ }^{3} \mathrm{He}$ pinning occurring at lower $T$ with a large strain $\left(10^{-7}\right)$ than with a low strain $\left(10^{-9}\right)$. In contrast, the phonon damping is independent of the strain amplitude.

Figure 5 shows additional measurements made on $\mathrm{X} 4$, a type 1 crystal that was cooled down under high strain $\left(10^{-7}\right)$ at $3 \mathrm{kHz}$ in order to expel all ${ }^{3} \mathrm{He}$ impurities into the liquid. Its coefficient $c_{44}$ is reduced by $80 \%$ even at $20 \mathrm{mK}$. This value is stable in time $\left({ }^{3} \mathrm{He}\right.$ stay trapped in the 


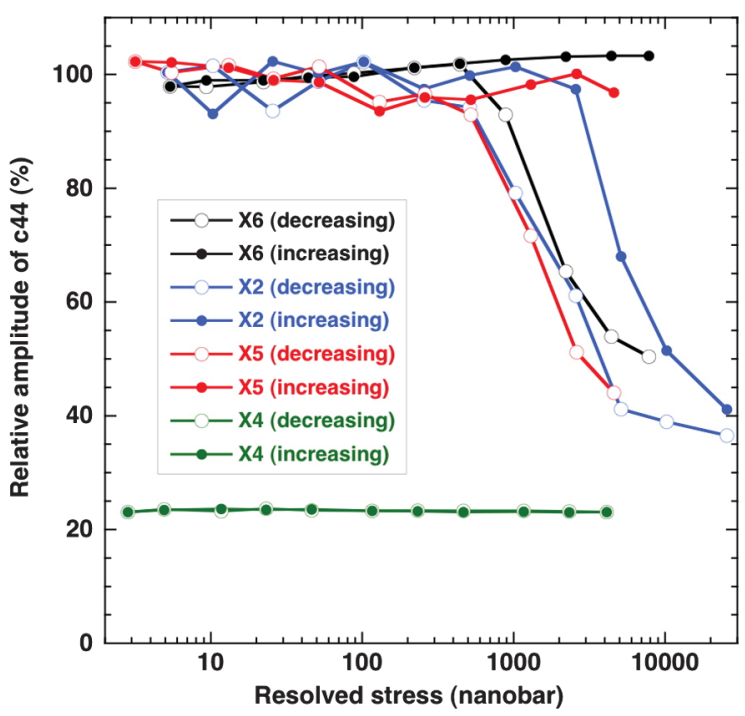

FIG. 5 (color). The relative amplitude of $c_{44}$ for 4 different crystals at $20 \mathrm{mK}$ as a function of the stress projected on the basal plane ("resolved"). At a threshold stress around $1 \mu$ bar, dislocations break away from ${ }^{3} \mathrm{He}$ impurities $[6,14]$. This threshold is larger when increasing the stress than when decreasing it. Being free of impurities, X4 shows a reduction of $c_{44}$ by $80 \%$, independent of stress.

liquid), and independent of the strain amplitude. After assuming that dislocations glide in the basal plane, Rojas et al. [9] found $86 \%$ reduction for a type 1 crystal of probably better quality (see Supplemental Material [10]). Here we demonstrate that this assumption was correct, and that the plasticity is very large in particular directions. We have not yet prepared a crystal with zero dislocation as might be possible [19]. Dislocations arranged in a simple Frank network should lead to less than $5 \%$ reduction in $c_{44}$ [20]. In our case, they may be aligned in sub-boundaries [21] where they cooperate to reduce $c_{44}$ much more. Assuming a typical dislocation density [22] $\Lambda=$ $10^{4} \mathrm{~cm}^{-2}$, we could estimate the amplitude of their motion. With a $10 \mathrm{kHz}$ strain $\varepsilon=3 \times 10^{-8}$, we find that dislocations move a distance $d$ of order $\varepsilon / \Lambda b=1 \mu \mathrm{m}$ corresponding to $6 \mathrm{~cm} / \mathrm{s}(b=3 \AA$ is the Burgers vector amplitude). This is 200 million Burgers vectors per second, a giant effect with no equivalent in classical crystals (see Supplemental Material [10]) [20,23,24].

In summary, we have demonstrated that the plasticity of ${ }^{4} \mathrm{He}$ crystals is reversible and anisotropic, due to the free gliding of dislocations parallel to the basal planes. Classical plasticity is irreversible because dislocations jump from one pinning site to another and multiply, leading to "work hardening." In our case, applying large stresses at low $T$ produces defects, which harden the crystals and are probably jogs because annealing at $1 \mathrm{~K}$ restores the high crystal quality (see Supplemental Material [10]). For simplicity, we show measurements in this article, which are recorded on cooling after annealing.
The giant plasticity of ${ }^{4} \mathrm{He}$ crystals is mainly due to their absolute purity at very low temperature. An interesting question is whether dislocations move by tunnelling or by thermal activation over Peierls barriers. Tunneling is suggested by the reversible motion at low $T$ without dissipation or strain dependence. However, quantum fluctuations probably reduce the kink energy $E_{k}$ by enlarging their width. If $k_{B} T>E_{k}$, kinks are not relevant, as is the case for surface steps $[25,26]$ whose dynamics is also dissipation free in the low $T$ limit. A calculation of $E_{k}$ could indicate if the dislocation motion is quantum or classical thanks to a negligible kink energy.

This work was supported by Grants from ERC (AdG 247258) and from NSERC Canada.

[1] R. A. Vardanian, Crystal Lattice Defects and Dislocation Dynamics (Nova Science Pub. Inc., New York, 2000).

[2] R. F. Tinder and J. Washburn, Acta Metall. 12, 129 (1964).

[3] A. Berghezan, A. Fourdeux, and S. Amelinckx, Acta Metall. 9, 464 (1961).

[4] D. Hull and D. J. Bacon, Introduction to Dislocations (Butterworth-Heinemann Elsevier, Oxford, 2000).

[5] L. Proville, D. Rodney, and M. C. Marinica, Nat. Mater. 11, 845 (2012).

[6] J. Day and J. Beamish, Nature (London) 450, 853 (2007).

[7] S. Sasaki, F. Caupin, and S. Balibar, J. Low Temp. Phys. 153, 43 (2008).

[8] C. Pantalei, X. Rojas, D. O. Edwards, H. J. Maris, and S. Balibar, J. Low Temp. Phys. 159, 452 (2010).

[9] X. Rojas, A. Haziot, V. Bapst, S. Balibar, and H. J. Maris, Phys. Rev. Lett. 105, 145302 (2010).

[10] See Supplemental Material at http://link.aps.org/ supplemental/10.1103/PhysRevLett.110.035301 for details on the experimental cell, the transducer calibration, sample preparation, orientation dependence, measurement protocol, and comparison with classical crystals.

[11] S. Balibar, A. Y. Parshin, and H. Alles, Rev. Mod. Phys. 77, 317 (2005).

[12] O. Syshchenko, J. Day, and J. Beamish, Phys. Rev. Lett. 104, 195301 (2010).

[13] I. Iwasa, K. Araki, and H. Suzuki, J. Phys. Soc. Jpn. 46, 1119 (1979).

[14] J. Day and J. R. Beamish, J. Low Temp. Phys. 166, 33 (2012).

[15] R. H. Crepeau, O. Heybey, D. Lee, and S. Strauss, Phys. Rev. A 3, 1162 (1971).

[16] D. S. Greywall, Phys. Rev. B 16, 5127 (1977).

[17] H. J. Maris and S. Balibar, J. Low Temp. Phys. 160, 5 (2010).

[18] I. Iwasa and H. Suzuki, J. Phys. Soc. Jpn. 49, 1722 (1980).

[19] J. P. Ruutu, P. Hakonen, A. Babkin, A. Parshin, J. Penttilä, J. Saramäki, and G. Tvalashvili, Phys. Rev. Lett. 76, 4187 (1996).

[20] J. Friedel, Dislocations (Pergamon, New York, 1964).

[21] I. Iwasa, H. Suzuki, T. Suzuki, and T. Nakajima, J. Low Temp. Phys. 100, 147 (1995). 
[22] O. Syshchenko and J. Beamish, J. Low Temp. Phys. 150, 276 (2008).

[23] A. Hikata and C. Elbaum, Phys. Rev. Lett. 54, 2418 (1985).

[24] R. J. M. Farla, I. Jackson, J. D. Fitz Gerald, U. H. Faul, and M. E. Zimmerman, Science 336, 332 (2012).
[25] P. Nozières, Eur. Phys. J. B 24, 383 (2001).

[26] E. Rolley, E. Chevalier, C. Guthmann, and S. Balibar, Phys. Rev. Lett. 72, 872 (1994); E. Rolley, C. Guthmann, E. Chevalier, and S. Balibar, J. Low Temp. Phys. 99, 851 (1995). 